\title{
A Prevalence Survey of Pediatric Type 2 Diabetes Mellitus Among Patients of Physicians in the Philippines
}

\author{
Evangeline P Costelo ${ }^{1}$, Gerry H Tan ${ }^{2 *}$, Lisa Marie Saldanha ${ }^{3}$ and Karen Wai ${ }^{3}$ \\ ${ }^{1}$ Department of Internal Medicine, Cebu Doctors University Hospital, Cebu City, Philippines
}

${ }^{2}$ Division of Endocrinology, Diabetes and Metabolism, Department of Internal Medicine, Cebu Doctors University Hospital, Cebu City Philippines

${ }^{3}$ Integrated Site Services, Quintiles East Asia Pte Ltd, Singapore

*Corresponding author: Gerry H Tan, Division of Endocrinology, Diabetes and Metabolism, Department of Internal Medicine, Cebu Doctors University College of Medicine, CDUH, Cebu City Philippines

\begin{abstract}
Objective: The primary objective of the study was to determine the prevalence of pediatric type 2 diabetes mellitus among patients of Filipino physicians.

Methods: A cross-sectional survey conducted from January to March 2014 among physician-members of the American Association of Clinical Endocrinologists-Philippine Chapter, Diabetes Philippines, Philippine Society of Endocrinology and Metabolism and Philippine Society of Pediatric Metabolism \& Endocrinology.

Results: The prevalence of pediatric type 2 diabetes mellitus among patients of respondents was $0.091 \%$ with all patients aged 10 to 18 years old. More than half were symptomatic and most of them were managed by adult endocrinologists and adult diabetologists. $73 \%$ of the patients used metformin as their initial drug of choice.
\end{abstract}

Conclusion: There was a low prevalence of pediatric type 2 diabetes mellitus among patients of Filipino physicians.

Keywords: Prevalence; Pediatric type 2 diabetes

\section{Introduction}

The global prevalence of type 2 diabetes among the pediatric age group has significantly increased over the last 10 years which could be attributable to the growing problem of childhood obesity. Culturally, Filipino parents associate 'fat' or 'overweight' children as being healthier than the children with appropriate for age weight. With the increasing prevalence of type 2 diabetes among the younger adult Filipino population and among the working class of Filipinos, there is a need to look into the prevalence of type 2 diabetes mellitus among the pediatric age group. The Philippine DALY (disability adjusted life years) of diabetes taken from an analysis of Institute for Health Metrics and Evaluation (IHME) for 1990 to 2010 showed an increased of the DALY of more than $200 \%$ [1]. This also showed the increasing burden of diabetes. The prevalence rate of type 2 diabetes mellitus among the adult Filipino population is $9.7 \%$ and the prevalence rate of impaired glucose tolerance is $12.5 \%$, but what is the prevalence of type 2 diabetes mellitus among the Filipino youth? [2, 3] The primary objective of the study was to determine the prevalence of pediatric type 2 diabetes mellitus among patients of Filipino physicians. The secondary objectives were to determine the medical specialization of the physician-respondents, their practice settings, patient treatment pathway from diagnosis to long term management and the most common treatment regimen given to pediatric type 2 diabetic patients.

\section{Methodology}

This is a cross-sectional survey conducted from January to March 2014. A validated survey questionnaire was distributed among physician-members of the American Association of Clinical Endocrinologists-Philippine Chapter, Diabetes Philippines, Philippine Society of Endocrinology and Metabolism and Philippine Society of Pediatric Metabolism \& Endocrinology. The survey questionnaire was reviewed for content by a team of Quintiles' representatives with expertise in clinical study feasibility, pediatric 
medicine and diabetology. The validation of the questionnaire was done by 3 member-physicians and their replies were not included in the final results. The survey questionnaire contained closedended questions to allow quantitative and qualitative results. The questions required "yes" or "no" type responses and number value responses.

The sample size was calculated at 95\% level of confidence and the maximum tolerable error for the prevalence estimate was 0.05 and using the overall prevalence rate from the SEARCH for Diabetes in Youth Study Group which was 0.22 cases per 1000 youth [4]. Respondent driven sampling (RDS) was then utilized to obtain the prevalence of pediatric type 2 diabetes mellitus among patients of Filipino physicians [5]. It was estimated that the survey should obtain at least 95 physician-respondents (representing 20\% of the physician-members of the diabetes-physician organizations) to obtain the sample size of 34 pediatric type 2 diabetes patients. A total of 120 English language survey questionnaires were distributed either through email or through mail. In March 2014, the responses from the completed survey forms were entered into Survey Methods, a web-based central repository [6]. Appropriate follow-ups were conducted to encourage the return of completed surveys. Data analysis was performed in April 2014 using Survey Methods.

\section{Results}

There were 106 (88.3\%) completed questionnaires received from the physician-members of the American Association of Clinical Endocrinologists-Philippine Chapter, Diabetes Philippines, Philippine Society of Endocrinology and Metabolism and Philippine Pediatric Society of Endocrinology and Metabolism. The field of specialization and the clinical practice setting of the respondents were Captured. The 106 respondents were seeing a total of 395,208 patients and about $71 \%(281,398)$ were type 2 diabetic patients. About 64\% (61) of them had patients with pediatric type 2 diabetic patients (under 18-year old). The prevalence of pediatric type 2 diabetes mellitus among patients of respondents was $0.091 \%$. The physician who initially diagnosed the patient as pediatric type 2 diabetic is the physician who would manage the condition of the patient. There were only $4(6.55 \%)$ respondents out of 61 respondents who would refer the pediatric type 2 DM patient to another specialist. These 4 respondents had their practice setting in a general hospital - private (tertiary hospital) and there was an available pediatric endocrinologist in their setting.

\section{Discussion}

Pediatric type 2 diabetes mellitus is defined as children and adolescents with type 2 diabetes mellitus and less than 18 years of age [7]. There are 442 physician members of the different diabetesphysician organizations in the Philippines. Of the 442 memberphysicians, the study obtained completed surveys from $24 \%$ of the member-physicians including 11 responses from the 17 members of the Philippine Society of Pediatric Metabolism \& Endocrinology. The prevalence of pediatric type 2 diabetes mellitus among Filipino patients of physician-respondents was $0.091 \%$ or 0.91 per 1000 patients of the physician-respondents. This is a higher prevalence rate compared to the total prevalence rate in SEARCH study which was 0.22 per 1000 . The SEARCH study also published a prevalence of 0.52 per 1000 youth aged 10 to 19 years with type 2 diabetes mellitus [4]. The SEARCH study was a community-based study conducted in the Unites States to describe the clinical characteristics, incidence, and prevalence of diabetes among Asian, Pacific Islander, and mixed Asian-Pacific Islander youth. The Philippines prevalence rate was also higher than the data obtained from the First UK survey of pediatric type 2 diabetes and MODY, a cross-sectional postal questionnaire survey of all consultants involved in the care of children with diabetes in the United Kingdom [8]. The crude minimum prevalence of type 2 diabetes under 16 years in the UK was 0.21 per 100,000 .

This study is not a community-based survey and hence might have underestimated the prevalence of pediatric type 2 diabetes mellitus. The study only looked at the patient population of physicians who were managing type 2 diabetes mellitus and determined the prevalence of pediatric patients with a diagnosis of type $2 \mathrm{DM}$. In the Philippines, most pediatric type 2 diabetes patients were managed by adult endocrinologists and adult diabetologists. Only a few were managed by pediatric endocrinologists. There are few pediatric endocrinologists in the Philippines and most of them practice in large tertiary hospitals in urban areas. This explains why most of the pediatric type 2 diabetics were managed by the adult endocrinologists and diabetologists. The age distribution of Filipino pediatric type 2 diabetes was the same as most of the available studies from US and Asia. All the patients were 10 years old to less than 18 years old with more patients seen among the $>15$ to < 18 years old age group (65.74\%) than with $>10$ to $<15$ years old (34.26\%). In the study, First UK survey of pediatric type 2 diabetes and MODY, the median age of pediatric type 2 DM in UK was 12.8 (3.7-15.9) [9]. Pediatric type 2 diabetes usually present among overweight adolescent patients with family history of type 2 DM. More than half of patients seen were symptomatic with polyuria, polydipsia and polyphagia while the other half were asymptomatic and blood sugar screening was done because of family history of type 2 diabetes and/or the patient was obese.

Most of the respondent physicians prescribed metformin as the first line of treatment as well as maintenance therapy for pediatric type 2 diabetes patients. In the Philippines, only Metformin and Insulin have been approved as pharmacologic therapies for pediatric type 2 diabetes. In the clinical practice guidelines for pediatric type 2 diabetes, the American Academy of Pediatrics has recommended clinicians must ensure that insulin therapy is initiated for children and adolescents with T2DM who are ketotic or in diabetic ketoacidosis and in whom the distinction between T1DM and T2DM is unclear and for these patients, insulin therapy should be initiated [10]. The clinical practice guidelines also state that in all other instances, clinicians should initiate a lifestyle modification program, including nutrition and physical activity, and start metformin as first-line therapy for children and adolescents at the time of diagnosis of T2DM. 


\section{Conclusion}

There is an alarming increase in the prevalence of Type 2 Diabetes in the Philippines [11]. Our study shows that the prevalence of Filipino pediatric type 2 diabetes mellitus among patients of Filipino physicians is low.

\section{Acknowledgement}

The authors wished to thank Adeline Sng and Jessica Lee for their assistance for data encoding. This paper was presented in part at the Annual meeting of the American Association of Clinical Endocrinologists (AACE) Annual Meeting in Nashville, Tennessee, May 2015. This research is partially funded by the AACE Philippines Research Advocacy.

\section{References}

1. (2013) Institute for Health Metrics Evaluation. What are the health challenges and successes in countries around the world?

2. Jimeno C, Sobrepeña L, Mirasol R (2012) DiabCare 2008: Survey on Glycaemic Control and the Status of Diabetes Care and Complications Among Patients with Type 2 Diabetes Mellitus in the Philippines. Philippine Journal of Internal Medicine 50(1): 15-22.

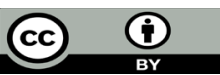

This work is licensed under Creative Commons Attribution 4.0 License

To Submit Your Article Click Here: Submit Article

DOI: $10.32474 / A D 0.2020 .02 .000145$
3. NHS (2013) $7^{\text {th }}$ Nutritional and Health Survey: 2008.

4. Dabelea D, Bell RA, D Agostino RB, Imperatore G, Johansen JM, et al. (2007). The incidence of diabetes in youth in the United States. JAMA 297(24): 2716-2724.

5. Johnston L, Sabin K (2010) Sampling hard-to-reach populations with respondent driven sampling. Methodological Innovations Online 5(2): 38-48.

6. Survey Methods (2014) https://www.surveymethods.com/index.aspx.

7. Kenneth C Copeland, Janet Silverstein, Kelly R Moore, Greg E Prazar, Terry Raymer, et al. (2013) Management of Newly Diagnosed Type 2 Diabetes Mellitus (T2DM) in Children and Adolescents. Pediatrics 131(2): 364-382.

8. Ehtisham S, Hattersley A, Dunger D, Barrett T (2004) First UK survey of pediatric type 2 diabetes and MODY. Arch Dis Child 89 (6): 526-529.

9. Goran M, Jaimie Davis, Louise Kelly, Gabriel Shaibi, Donna Spruijt Metz, et al. (2008) Low Prevalence of Pediatric Type 2 Diabetes: Where's the Epidemic? J Pediatr 152(6): 753-755.

10. Aanstoot HJ, Anderson BJ, Daneman D, Danne T, Donaghue K, et al. (2007) The Global Burden of Youth Diabetes: Perspectives and Potential. Pediatric Diabetes. 8 (Suppl 8): 1-44.

11. Tan GH (2015) Diabetes Care in the Philippines. Annals of Global Health 81(6): 863-869.

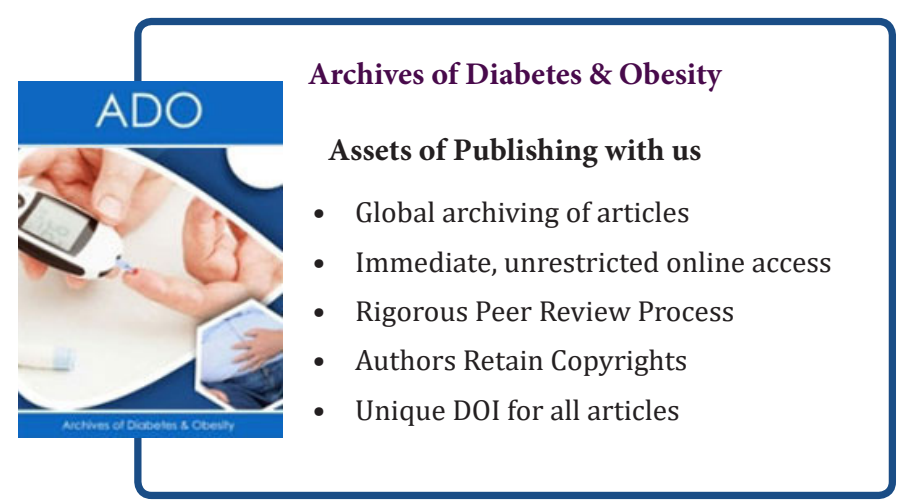

